\section{MS1-P16 Recent results and developments on PROXIMA 2A}

William Shepard ${ }^{1}$, Denis Duran ${ }^{1}$, Sebastian Le Couster ${ }^{1}$, Frederic Blache $^{1}$, Aurelien Delmotte ${ }^{1}$, Roger Fourme ${ }^{1}$, Gavin Fox ${ }^{1}$, Rob Meijers ${ }^{1}$, Thierry Moreno ${ }^{1}$, Sandra Pierre-Joseph ${ }^{1}$, Martin Savko

1. Synchrotron SOLEIL

email: william.shepard@synchrotron-soleil.fr

PROXIMA 2A is an energy tunable $(6-15 \mathrm{keV})$ micro-focus beamline at Synchrotron SOLEIL dedicated to macromolecular crystallography (MX). Since opening in March 2013, many users have benefited from its fine focus $\left(10 \mu \mathrm{m} \times 5 \mu \mathrm{m}, \mathrm{H} \times \mathrm{V}\right.$ FWHM) and high flux $\left(>10^{12}\right.$ $\mathrm{ph} / \mathrm{s}$ ), and with such a high flux density, they have discovered that their micro-crystals diffract beyond their expectations. The experiments are controlled via the MXCuBE_V2 interface [1], which not only launches standard and MAD data collections, but also records and manages multiple positions on the sample. The micro-focused X-rays are frequently exploited in order to select out the best diffracting zone of a crystal. With this mind, grid and line scans are currently automated via command line, and these functionalities will be soon incorporated within the MXCuBE interface. Helical scans are already available, implemented within MXCuBE and frequently employed by the users. The data quality from helical scans is comparable to standard data collection strategies. The beamline is also equipped with a CATS robotic sample changer, which consists of a 9-unipuck dewar (total 144 pins). Up to 200 samples have been screened in a 24-hour period. The CATS robot is also capable of screening crystallization plates, and although this functionality is still being commissioned, the preliminary results are very encouraging. Developments are also underway with other in situ data collection strategies. In particular, the beamline is equipped with a Plugmaker, which is a device that can perform crystallization experiments on micro-fluidic chips. These chips can be mounted directly on to the goniometer and the crystals tested under X-rays. Finally, the current area detector (ADSC Q315r) will be soon upgraded to an EIGER 9M, which will offer a faster readout and lower noise level. Coupled with its high flux density and multiple functionalities, PROXIMA 2A has become a versatile instrument for the MX community.

[1] Gabadinho et al., J. Synchrotron Rad. 2010, 17, 700-707.

Keywords: beamline, microcrystallography, macromolecular crystallography, synchrotron

\section{MS1-P17 VMXm: a new sub-micron beamline for macromolecular crystallography at Diamond Light Source}

Jose Trincao ${ }^{1}$, Anna Warren ${ }^{1}$, Pierre Aller ${ }^{1}$, Graham Duller ${ }^{1}$, Kevin Wilkinson ${ }^{1}$, Andy Stallwood ${ }^{1}$, David Laundy ${ }^{1}$, Lucia Allianeli ${ }^{1}$, Kawal Sahwney ${ }^{1}$, Guenther Rehm ${ }^{1}$, Gwyndaf Evans ${ }^{1}$

1. Diamond Light Source, Diamond House, Harwell Science and Innovation Campus, Didcot, Oxfordshire, OX11 ODE,UK

\section{email: jose.trincao@diamond.ac.uk}

The increasing complexity of currently studied biological targets has pushed the demand for microfocus MX beamlines with ever smaller beam sizes and higher intensities. Serial Femtosecond Crystallography at XFELs is one approach to sub-micron crystals, but is still largely inaccessible to most users and far from ready for mainstream usage. VMXm is a new beamline being built at Diamond Light Source aiming to bridge the gap between current microfocus beamlines and XFELs. It is being designed to optimize the data to protein-material ratio in microcrystallography targeting crystal sizes down to 0.5 microns. The beamline optics will deliver a beamsize of $0.5->5 \mu \mathrm{m}$ vertically, using a single custom-profiled fixed focal length mirror [1], and $0.5-5$ um horizontally via a two stage demagnification scheme and a variable secondary source aperture. The beamline will operate at wavelengths between 7 and $25 \mathrm{keV}$ delivering between $10^{12}$ and $10^{13} \mathrm{ph} / \mathrm{s}$ to the sample (at 12 $\mathrm{keV}$ ) depending on the optical configuration. The source, optics and end-station designs for VMXm will be presented together with novel solutions to the problem of sample visualisation and alignment for submicron crystals.

\section{References}

[1] Laundy, D., Alianelli, L., Sutter, J., Evans, G. \& Sawhney, K. Surface profiling of X-ray mirrors for shaping focused beams. Optics Express 23, 1576-1584 (2015).

Keywords: micro-focus, VMXm, beamline, sub-micron, serial crystallography 